\title{
Druggable vitiligo genome: a fast track approach to take the genome wide association to the clinic
}

\begin{abstract}
Vitiligo, a skin depigmentation disorder, affects 0.5 to $1 \%$ of the population around the globe. While not life threatening, the disorder is associated with serious psychological trauma. Currently no known cure is available and the precise etiology is unknown. Genome-Wide Association Studies (GWAS) may provide clues to better understanding the disorder for the development of novel therapeutics. Mining the GWAS databases resulted in the identification of 51 Vitiligo-associated genes (VAG) encompassing protein-coding sequences, noncoding RNAs and pseudogenes. A druggable class of proteins including enzymes, transporters, transcription factors and secretome products was part of the VAG. The VAG were also genetically linked to autoimmune, cancer, cardiovascular, inflammation, infections and neurological diseases. Two genes, Interleukin 2 receptor alpha (IL2RA) and Tyrosinase (TYR) are FDA approved targets. Unique population-specific genes were identified in the GWAS databases. Chemogenomics approaches identified 246 compounds targeting the VAG. Key pathways involving the VAG's mechanism included apoptosis, endocrine, immune, infection, metabolic, neuronal and transcription factor signaling. Five lead targets, four enzymes and one transporter, emerged from this study with bioactive drug-like compounds $(<100 \mathrm{nM})$. The VAG were linked to diverse environmental factors including antioxidants, DNA damage, oxidative stress and UV. Drug bank compounds encompassing anti-infectives, anti-neoplastics, immune modulators and nutraceuticals/ supplements were identified as targets for the VAG. The FDA approved drugs from the VAG studies can be repurposed and off label use can be developed for the treatment of Vitiligo.
\end{abstract}

Keywords: chemogenomics, chemoinformatics, druggable genes, human genome, ligand binding, protein $3 \mathrm{~d}$ structures, response to therapy, skin disorders
Volume 2 Issue 3 - 2015

\author{
Ramaswamy Narayanan \\ Department of Biological Sciences, Charles E. Schmidt College \\ of Science, Florida Atlantic University, USA
}

Correspondence: Ramaswamy Narayanan, Department of Biological Sciences, Charles E. Schmidt College of Science, Florida Atlantic University, 777 Glades Road, Boca Raton, FL 33431, USA, Tel + |56|2972247, Fax + |56|2973859, Email rnarayan@fau.edu

Received: May 28, 2015 | Published: July 24, 2015
Abbreviations: canSAR, integrated cancer drug discovery platform; chEMBL, database of bioactive compounds at the european bioinformatics institute of the european molecular biology laboratory (EMBL); FDA, us federal drug administration; GWAS, genome-wide association studies; VAG, vitiligo-associated genes; PDB, protein database; RO5, rule of five

\section{Introduction}

Vitiligo is an acquired depigmenting disorder of the skin and the mucous membrane characterized by loss of melanocytes, exocrine cells from the basal layer of the Epidermis and the matrix portion of the hair bulb. ${ }^{1,2}$ The disease is seen at a frequency of $1 \%$ of the world population. ${ }^{3}$ Vitiligo is classified into four types based on the distribution of the hypopigmented lesions: non-segmental Vitiligo (NSV), segmental Vitiligo (SV), mixed NSV and SV and unclassified types including focal, multifocal asymmetrical non-segmental and mucosal at one site. ${ }^{2}$ The NSV subtypes include focal at onset, mucosal, acrofacial, generalized and universal. Generalized Vitiligo occurs later in life at sites sensitive to pressure and is often progressive with flare-ups. SV usually begins in childhood most commonly in the face and remains stable. ${ }^{3}$ The white skin patches associated with Vitiligo have been recognized over thousands of years. ${ }^{4}$ Despite the long history, the etiology remains unknown. In the recent years, numerous theories have been put forward. ${ }^{1,5-12}$ Current evidence encompasses four main theories: the autoimmune hypothesis, the neural hypothesis, the self destruct hypothesis and the growth factor defect hypothesis. ${ }^{13}$ However, none of these have been conclusively proven to date. It is highly likely that Vitiligo is a polygenic trait and a convergent theory, combining elements of different theories across a spectrum of expression, is the most accurate etiology. ${ }^{7}$ The association of Vitiligo with other known autoimmune disorders such as Addison's Disease, Hashimoto's Thyroiditis, Pernicious Anemia and Alopecia Areata lend credence to the autoimmune theory of disease. ${ }^{14}$ While non-life threatening, the disfigurement associated with Vitiligo causes serious emotional stress and depression and interferes with the quality of life..$^{15,16}$ No known cure is available and current treatment options are limited. Diverse therapeutic modalities are in current use, including topical (corticosteroids, vitamin D derivatives, calcinurin inhibitors, prostaglandin E 2, Pseudocatalase), phototherapy [psoralen plus UV-A (PUVA), psorolen with sun light (PUVAsol), surgical techniques, Excimer laser, and combinations of topical therapy with light treatment. ${ }^{1,17-19}$ Other medicines being tried include alternative medicines such as ginkgo biloba, ${ }^{20,21}$ topical fluorouracil, ${ }^{22}$ minioxidil, ${ }^{23}$ oral L-phenylalanine, ${ }^{24}$ homeopathy and aurvedic medicine ${ }^{17,25}$ and depigmentation with monobenzyl ether. ${ }^{26}$ The success rate with all of these drugs is variable and uncertain. At the present time the only FDA approved treatment is the use of Excimer laser (308nm). ${ }^{27,28}$ Currently, even if the treatment is efficacious, often the depigmentation recurs. A maintenance therapy is often required. ${ }^{19}$ In view of the large number of people affected around the globe by Vitiligo, novel therapeutic approaches as well biomarkers for identifying at risk individuals are urgently needed. 
Currently 53 clinical trials are underway for the treatment of Vitiligo (clinicaltrials.gov). The International Clinical Trial Registry (WHO) platform records 28 clinical trial records for Vitiligo. The European Union Clinical Trials Register shows 11 clinical trial records currently underway in Europe. These trials largely revolve around light treatment, excimer laser, topical steroids and calcineurin inhibitors, statins, anti oxidants, vitamins, ginkgo biloba supplements, skin grafts, melanocytes and keratinocytes transplantation. The GWAS dataset for the Vitiligo-associated phenotypes are becoming available. ${ }^{29-34}$ Reasoning that the phenotypic polymorphic traits associated with the Vitiligo genes might provide an attractive starting point for drug targets discovery, the GWAS databases were mined for VAG. Fifty-one genes were identified from these studies encompassing druggable targets. Chemogenomics approaches identified highly active small molecular weight compounds $(<100 \mathrm{nM})$ for five of these targets, which include enzymes and a transporter. FDA approved and experimental drugs as well as nutraceuticals were identified targeting the VAG. Repurposing these approved drugs may offer a therapeutic benefit to the Vitiligo patients.

\section{Materials and methods}

The bioinformatics and proteomics tools used in the study have been described. ${ }^{35-37}$ The GWAS catalogue ${ }^{38}$ and the NCBI PhenomeGenome Integrator, PheGenI ${ }^{39}$ were used to create a working list of VAG. Characterization of the VAG was performed using the GeneALaCart, GeneAnalytics and VarElect data mining tools from the GeneCards. ${ }^{40}$ The VarElect is a Variant Election application for disease/phenotype-dependent gene variant prioritization. VarElect combines information within the LifeMap Knowledgebase of GeneCards, MalaCards, the human disease database, LifeMap Discovery, the regenerative medicine, and PathCards, the unified human biological pathways database. In addition, Gene Analytics Powered by LifeMap's Gene Cards Suite integrated knowledgebase, which utilizes data from $>120$ select sources was also used to batch analyze the VAG for gene expression-based analysis. Comprehensive background information on the VAG was obtained by using the GeneALaCart tool from the GeneCards for Gene Ontology, super pathways and drug bank compounds. The protein annotation and chemical structure-based mining was performed using the canSAR 2.0 integrated knowledgebase, a publically available database..$^{41,42}$ The browse canSAR section was used and the Vitiligo-associated proteins were batch analyzed for protein annotations, 3D structures, compounds and bioactivity details. The protein 3D structure template models-related information was obtained from the Swiss Protein Database. The chemical structures were obtained from the chEMBL. ${ }^{4}$ Comprehensive gene annotation for the VAG was established using the GeneCards, ${ }^{40}$ the DAVID functional annotation tool ${ }^{44}$ and the UniProt $^{45}$ databases. FDA approved drug related information was obtained from the Drug-Bank. ${ }^{46}$ Protein expression was verified using the Human Protein Map, ${ }^{47}$ ProteomicsDB ${ }^{48}$ and the Multi Omics Protein Expression Database. ${ }^{49,50}$ The canSAR compounds link for genes has diverse filters such as activity and assay types, concentrations, molecular weight, $\mathrm{RO} 5$ violations, prediction of oral bioavailabilty and toxicophores. Putative drug hits were filtered from the canSAR datasets for the VAG-associated genes using Lipinski's rule of five (also known as Pfizer's rule of five), RO5. The RO5 is a rule of thumb to evaluate druggableness or to determine whether a compound with a certain pharmacological or biological activity possesses properties that would make it a likely orally active drug in humans. ${ }^{51-53}$ Highest stringency was chosen for the RO5 violation (value $=0$ ). Protein 3D structural homology was set at $100 \%$. The ligand-based druggability score was chosen at $>60 \%$ confidence. Drugs with IC50values $(<100 \mathrm{nM})$, inhibitory activities and Ki values were chosen for the canSAR output. Toxicophore negative was chosen to filter the hits for toxicity associated compound structures. ${ }^{54}$

\section{Results}

\section{Vitiligo-associated proteins from the GWAS studies}

The GWAS databases (the GWAS catalogue at European Molecular Biology Laboratory-European Bioinformatics Institute -EMBL-EBI) and the NCBI PheGenI were mined for the query term Vitiligo. The output from these two databases was merged and duplicate entries were removed. This resulted in the identification of 51 Vitiligo genes including protein coding genes, noncoding RNAs and pseudogenes (Figure 1 \& Supplemental Table S1). As a class, enzymes were the largest amongst the VAG (kinase, ligase, helicase, tyrosine phosphatase, granzyme B, caspase 7 and ribonuclease T2). Other druggable classes of proteins included transporters (Phospholipid, amino acid, nucleoside and Choline) and receptors (Mast cell immunoreceptor signal transducer, RIG-I-like receptor 2, Interleukin-2 receptor subunit alpha, Toll-interleukin-1 receptor domain-containing adapter protein and Suppressor of T-cell receptor signaling 2).

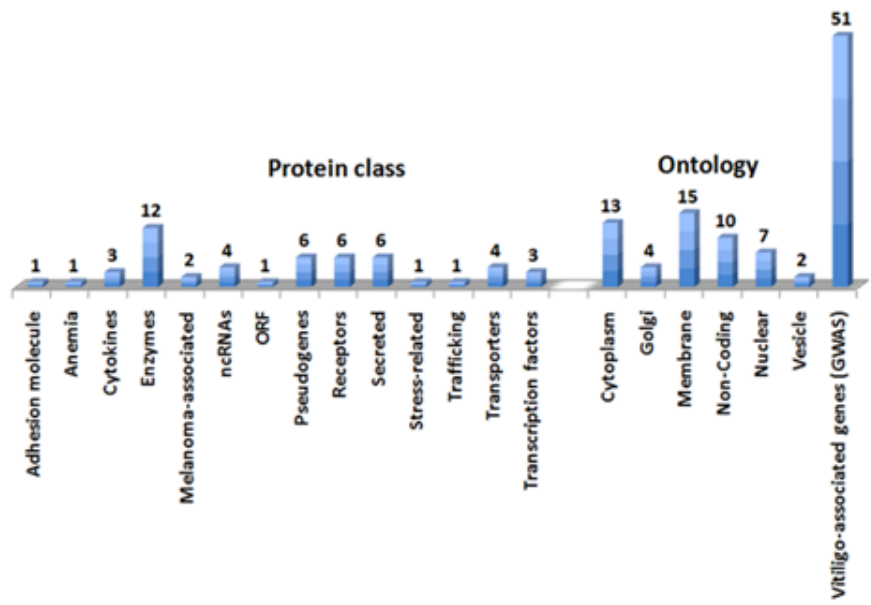

Figure I Vitiligo-Associated Genes: Characterization The VAG output from the GWAS datasets was analyzed by the GeneALacart Meta Analysis tool from the GeneCards for protein classes and Gene Ontology. The numbers above the bars indicate the number of genes in the indicated class. Abbreviations: GWAS: Genome-Wide Association Studies; ncRNAs: noncoding RNAs

Secreted proteins included Complement $\mathrm{C} 1 \mathrm{q}$ tumor necrosis factorrelated protein 6, Prolyl endopeptidase, Granzyme B, Ribonuclease T2, calcium-binding protein 2 and Thyroglobulin. Two of the Vitiligo proteins were associated with melanoma (Prolyl endopeptidase FAP and Interferon-induced helicase $\mathrm{C}$ domain-containing protein 1). The Gene Ontology analysis revealed distinct subcellular locations of the Vitiligo genes including cytoplasmic, nuclear, golgi, vesicular, secreted and membrane bound proteins (Figure 1 \& Supplemental Table S1).

\section{Vitiligo proteins in other diseases and population distribution}

Vitiligo is often seen in patients with autoimmune and inflammatory diseases. ${ }^{3,55}$ It is, however, unclear whether such an occurrence is a coincidence. In order to identify common pathways and possible links to diverse diseases and Vitiligo, the VAG were analyzed for phenomo-genome genetic association for disease-related variants using the NCBI PheGenI and VarElect datamining tool from the GeneCards (Figure 2, panel A). The VAG association was seen with autoimmune diseases, allergy, cancer, inflammation, infections and neurodegenerative diseases. The availability of the GWAS datasets 
from around the globe with the 1000 genome project ${ }^{56}$ enabled the study to expand into population relevance (Figure 2, panel B). Twenty-seven of the VAG were found to be risk factors for diverse diseases including skin diseases (Supplemental Table S1).
A

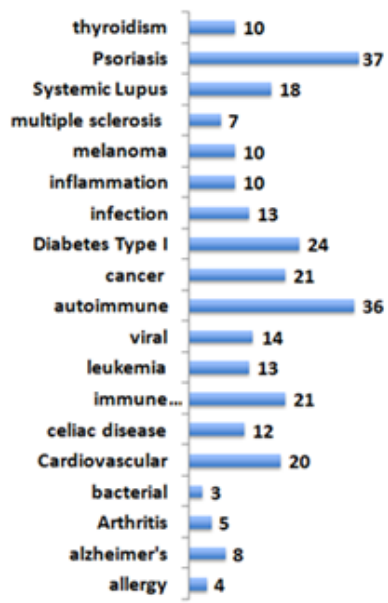

B

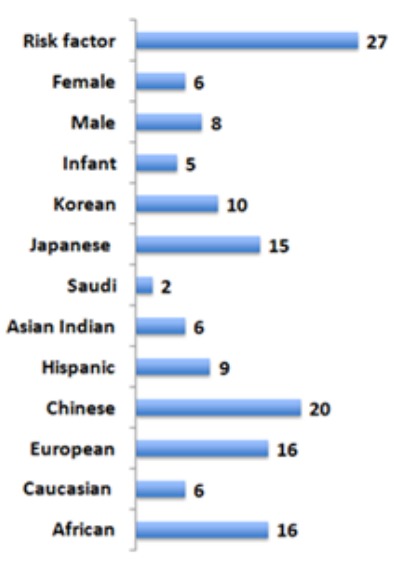

Figure 2 Vitiligo Genes: Other Diseases and Population Distribution The genetic association of the VAG with other diseases was analyzed using the NCBI PheGenl tool (Panel A). The polymorphic distribution of theVAG across the diverse human genome was analyzed using the Varelect Meta analysis tool from the GeneCards.

Distinct genes related single nucleotide polymorphisms (SNPs) were associated with unique population (Supplemental Table S1). These included patients from Japan (heterogeneous nuclear ribonucleoprotein A1 pseudogene 2|HNRNPA1P2), Africa (solute carrier family 44, member $4 \mid$ SLC44A4, zinc finger, MIZ-type containing 1|ZMIZ1) and Europe (potassium channel, subfamily $\mathrm{K}$, member $12 \mid \mathrm{KCNK} 12$, mitochondrial ribosomal protein $\mathrm{S} 17$ pseudogene 7| MRPS17P7, SPARC related modular calcium binding 2|SMOC2 and toll-like receptor adaptor molecule 1|TICAM1).

Association of some of the VAG is gender-specific: males (ATPase, aminophospholipid transporter, class I, type 8B, member 1 , ataxin 2 and RPGRIP1-like) and females (tyrosinase). Generalized Vitiligo and psoriasis shared 37 genes from the VAG list. Interestingly, an enzyme, ADP-ribosylarginine hydrolase, was found to be associated with segmented, but not generalized Vitiligo. Variants related to these unique genes may provide a Vitiligo fingerprint or biomarkers potential to monitor Vitiligo in a population specific manner.

\section{Vitiligo genes: types of mutations}

The Vitiligo genes were investigated for the types of mutations occurring in all tissues versus skin tissue (Figure 3). In the skin tissue germ line, pathogenic and causative mutations were seen for three genes 1) Fanconi anemia, complementation group A, 2) solute carrier family 29 (equilibrative nucleoside transporter), member 3 and 3) tyrosinase.

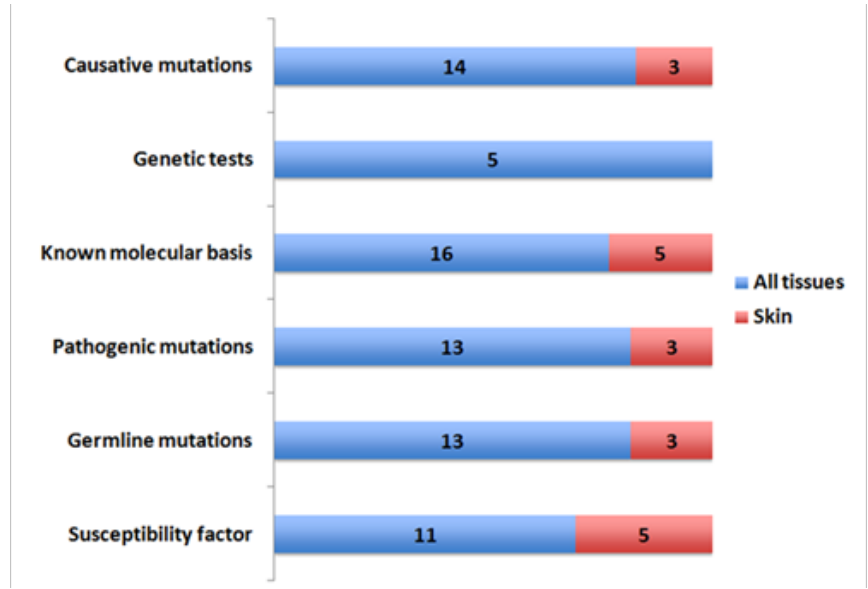

Figure 3 Mutational Classes of the Vitiligo Genes The mutational classes of the VAG analyzed by the VarElect Meta Analysis tool fro the GeneCards is shown (blue: all tissues, red: skin tissue). The numbers indicate the number of genes for the indicated class.

Five genes predictive of susceptibility to skin diseases/risk factors were also identified in these studies. These included granzyme B (granzyme 2, cytotoxic T-lymphocyte-associated serine esterase 1), major histocompatibility complex, class $1 \mathrm{~B}$, interferon induced with helicase $\mathrm{C}$ domain 1, protein tyrosine phosphatase, non-receptor type 22 (lymphoid) and fibroblast activation protein, alpha.

\section{Pathways mapping of the Vitiligo genes}

A Meta analysis tool, Gene Analytics, from the GeneCards was used to establish pathways implicated with the Vitiligo genes (Table 1). Consistent with the complex multifactorial etiology of Vitiligo, ${ }^{7}$ the VAG were found to be associated with pathways involving immune function, infectious diseases, endocrine function, apoptosis and survival, transcription factor signaling, metabolic and neural pathways. A group of genes common to these diverse pathways encompasses caspase 7, apoptosis-related cysteine peptidase, granzyme B (granzyme 2, cytotoxic T-lymphocyte-associated serine esterase 1), interleukin 2receptor, alpha and tyrosinase. Vitiligo is often associated with diverse environmental stress (e.g. DNA damage, stress, UV exposure). However, the precise role of any of these in the etiology of Vitiligo is unclear. Using the GeneAnalytics Meta Analysis tool, the variants of the VAG were analyzed for association with diverse factors (Supplemental Table S1). Distinct patterns of polymorphic variants were found to be associated with cell death (apoptosis, differentiation), steroids, stress-related, DNA damage (reactive oxygen, UV, phototherapy), melanocytes and vitamins and supplements.

Table I Pathway mapping of the vitiligo genes. Super Pathways involved with theVAG analyzed by the Gene Analytics Meta Analysis tool (GeneCards) are shown.

\begin{tabular}{ll}
\hline Super Pathways (Gene analytics) & Vitiligo-Associated Genes (VAG) \\
\hline Immune & \\
\hline Lymphocyte Signaling & BACH2, GZMB, IFIHI, IL2RA, PTPN22 \\
Allograft Rejection & CASP7, GZMB, HLA-B, IL2RA, TG \\
IL-I5 Signaling and Its Primary Biological Effects in Different Immune Cell Types & GZMB, IL2RA \\
ILI 2-mediated Signaling Events & GZMB, IL2RA \\
Downstream Signaling in Naive CD8+ T Cells & GZMB, IL2RA \\
\hline
\end{tabular}


Table Continued....

\begin{tabular}{|c|c|}
\hline Super Pathways (Gene analytics) & Vitiligo-Associated Genes (VAG) \\
\hline \multicolumn{2}{|l|}{ Infection } \\
\hline Influenza A & HLA-B, IFIHI, IL2RA, TICAMI \\
\hline Pertussis & CASP7,TICAMI \\
\hline \multicolumn{2}{|l|}{ Endocrine } \\
\hline Thyroxine (Thyroid Hormone) Production & TG \\
\hline \multicolumn{2}{|l|}{ Apoptosis } \\
\hline Activation of Caspases Through Apoptosome-mediated Cleavage & CASP7 \\
\hline Apoptosis and Survival Caspase Cascade & CASP7, GZMB \\
\hline Apoptosis (WikiPathways) & CASP7, GZMB \\
\hline Activation of $\mathrm{BH} 3$-only Proteins & CASP7, GZMB \\
\hline Granzyme Pathway & CASP7, GZMB \\
\hline \multicolumn{2}{|l|}{ Signaling } \\
\hline NF-kappaB Signaling & IFIHI,TICAMI \\
\hline S-IP Stimulated Signaling & CASP7, CDH23 \\
\hline \multicolumn{2}{|l|}{ Metabolic } \\
\hline Riboflavin Metabolism & TYR \\
\hline (S)-reticuline Biosynthesis II & TYR \\
\hline \multicolumn{2}{|l|}{ Neuronal } \\
\hline Dopamine Metabolism & TYR \\
\hline Neurotransmitter Uptake and Metabolism In Glial Cells & SLCIA2 \\
\hline Parkinsons Disease Pathway & ATXN2, CASP7 \\
\hline
\end{tabular}

\section{Vitiligo genes in the secretome}

The availability of the human proteome data sets ${ }^{47,48,57}$ allows the analysis of the disease-related genes in diverse body fluids. The human secretome encompasses proteins secreted by both the classical (signal peptide cleavage) and non-classical secretory pathways such as shed receptors and enzymes. ${ }^{58-61}$ Identifying the Vitiligo proteins secreted in the body fluids is crucial to developing a biomarker potential for the genes. Hence the proteomics database, the MOPED ${ }^{50}$ was batch analyzed for the 51 VAG (Figure $4 \&$ Supplemental Table S1). The VAG protein expression was detected in diverse body fluids including ascites, blood, cerebrospinal fluid, earwax, milk, pancreatic juice, saliva, semen and urine. The proteins detected in fluids such as 1) earwax (BTB and CNC homology 1, basic leucine zipper transcription factor 2|BACH2;DEAD (Asp-Glu-Ala-Asp) box helicase 6 |DDX6; Fanconi anemia, complementation group A FANCA; ribonuclease T2|RNASET2 and solute carrier family 44, member 4 |SLC44A4), 2) milk (HLA-B), saliva (major histocompatibility complex, class I, B|HLA-B and ribonuclease T2| RNASET2) and 3) urine (major histocompatibility complex, class I, B |HLA-B; arginine-glutamic acid dipeptide (RE) repeats |RERE) could provide a basis for non-invasive biomarker for prediction of risk as well as response to therapy.

\section{Chemogenomics of Vitiligo-associated genes}

In order to develop a drug therapy potential for the Vitiligo proteins, the 51 genes were analyzed using the CanSar protein annotation tool (Supplemental Table S1). Druggability of these genes was inferred from the $3 \mathrm{D}$ structures and the ligand binding scores ( $>70 \%$ confidence). Thirty-seven Vitiligo proteins were predicted to be druggable by the canSar annotation tool (Figure $5 \&$ Supplemental Table S1).
Among these hits, 18/37 proteins had a significant ligand binding druggability percentile rank $(>70 \%)$. These hit targets encompassed enzymes, transporters, receptors and secretome. Bioactive small molecular weight $(<500)$ compounds $(\mathrm{n}=363,<1 \mathrm{uM})$ were identified targeting the Vitiligo proteins. A significant number of these drug-like compounds were predicted to be non- toxic and orally bioavailable (no toxicophore, RO5 value - zero). Highly active compounds ( $\mathrm{n}=46$, $<10 \mathrm{nM}$ ) for three unique targets (Granzyme B|GZMB; HLA class II histocompatibility antigen|HLA-B and Tyrosine-protein phosphatase non-receptor type 22 (PTPN22) were identified.

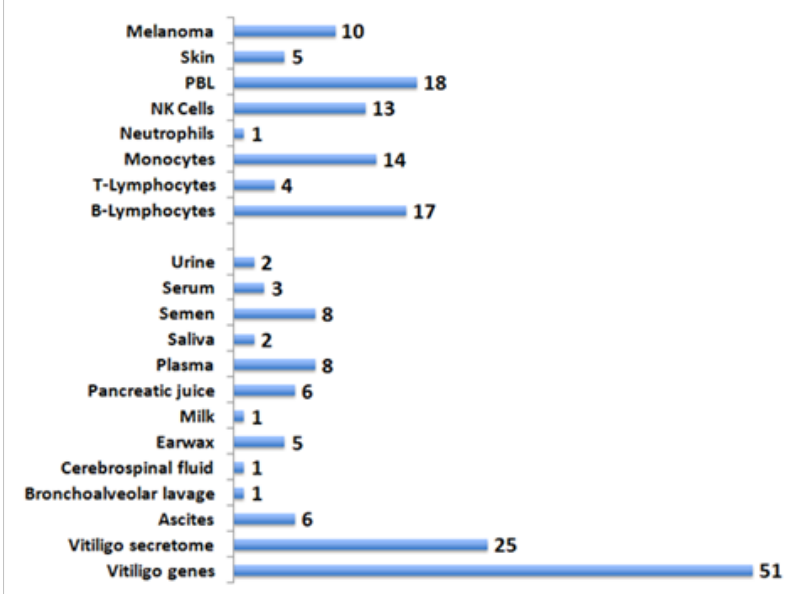

Figure 4 Mutational Classes of the Vitiligo Genes The mutational classes of the VAG analyzed by the VarElect Meta Analysis tool fro the GeneCards is shown (blue: all tissues, red: skin tissue). The numbers indicate the number of genes for the indicated class. 


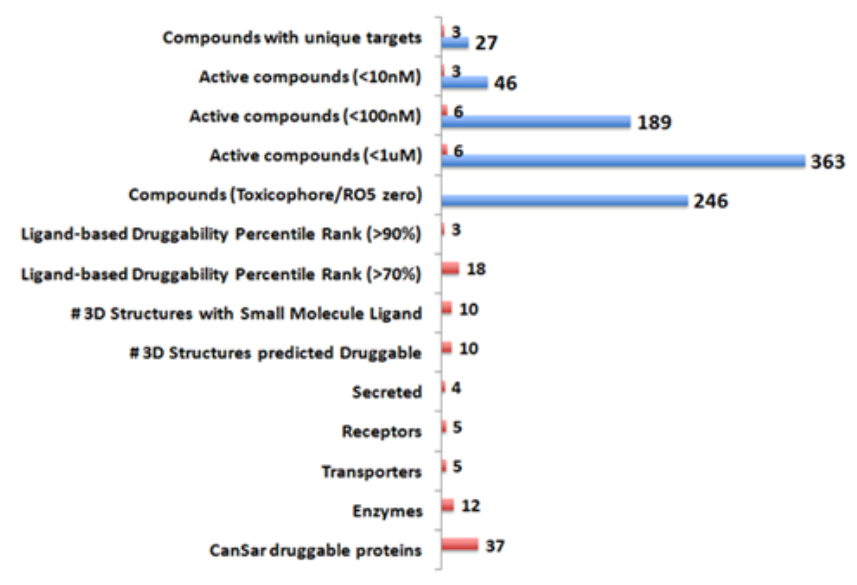

Figure 5 Chemogenomics of theVitiligo Genes The CanSar protein annotation tool was used to batch analyze the VAG. A summary of output based on the druggable 3D structures of the proteins and the ligand-based druggability indication is shown. The numbers indicate the number of proteins (red) and bioactive compounds (blue). Only active compounds for the proteins (<luM, $>90 \%$ confidence) are shown.

Abbreviations: $\mathrm{RO} 5$, rule of five

Five lead targets emerged with drug-like compounds $(<100 \mathrm{nM})$ bioactive in inhibitory assays (Supplemental Table 2). These leads included four enzymes (caspase 7-CASP7; fibroblast activation protein, alpha-FAP; granzyme B- GZMB and tyrosinase- TYR) and one transporter protein (solute carrier family 1 (glial high affinity glutamate transporter), member 2- SLC1A2). The five chEMBL compounds identified showed a high degree of selectivity to the target proteins. Two of these leads (chEMBL303944 and chEMBL24152) targeted unique enzymes, granzyme B |GZMB and Tyrosinase|TYR respectively.

\section{Drug bank compounds for the Vitiligo genes}

Repurposing existing FDA approved drugs for other indications is becoming increasingly attractive. ${ }^{62-64} \mathrm{~A}$ powerful example of this concept was recently demonstrated for the Ebola virus disease therapeutics. ${ }^{36,65}$ Association of genes with multiple diseases opens up avenues for expanding the repurposing strategy. Hence, the 51 Vitiligo genes were screened against the drug bank of compounds using the GeneALaCart Meta analysis tool of the GeneCards. The output was also compared against the FDA database for approved and experimental drugs, nutraceuticals and supplements (Phase I to Phase III). Additional hints of drug therapy use for the Vitiligo genes were obtained from the recently described proteomics reports encompassing putative drug therapy potential for the human proteome..$^{57,66}$ Two genes, Interleukin-2 receptor subunit alpha (immune suppression, transplant rejection and cancer) and tyrosinase (dermatology, acne, antimicrobial, melanocytes depigmentation, DNA synthesis inhibition), are FDA-approved drug targets (Table 3).

Table 2 Repurposing approved drugs for vitiligo therapy: Drug bank data.

\begin{tabular}{|c|c|c|}
\hline Vitiliogo associated genes (VAG) & Drugs (drug bank compounds) & Indications \\
\hline ADPRH, CASP7, GZMB, HLA-B, IL2RA, SLCIA2, TG, TYR & Aspartate & Nutraceutical \\
\hline ADPRH, CASP7, FANCA, HLA-B, IL2RA, SLCIA2, TG, TYR & $\mathrm{H} 2 \mathrm{O} 2$ & Infection|anticancer \\
\hline ADPRH, GZMB, HLA-B, IL2RA, PTPN22, SLCIA2, TG, TYR & Arginine & Nutraceutical |immune responsesl tissue repair \\
\hline ADPRH, CASP7, GZMB, IL2RA, PTPN22, SLCIA2, TG,TYR & Glutamate & nutritional supplementation \\
\hline ADPRH, CASP7, GZMB, HLA-B, SLCIA2,TG & Lactate & $\begin{array}{l}\text { dry, scaly skin (xerosis) and ichthyosis vulgaris and } \\
\text { for temporary relief of itching associated with these } \\
\text { conditions }\end{array}$ \\
\hline GZMB,TYR & Concanamycin A & osteoporosis|infections|anticancer \\
\hline ADPRH, HLA-B, IL2RA, TG, TYR & Polysaccharide & $\begin{array}{l}\text { Nutraceutical| treatment and prevention of } \\
\text { osteoarthritis, by itself or in combination with } \\
\text { chondroitin sulfate }\end{array}$ \\
\hline ADPRH,TG,TYR & Glucosamine & osteoarthritis|dietary supplement \\
\hline GZMB, IL2RA, TG & IVIG & $\begin{array}{l}\text { immunodeficiencies, as well as autoimmune and } \\
\text { inflammatory disorders. }\end{array}$ \\
\hline HLA-B, IL2RA & Phycoerythrin & Anticancer \\
\hline HLA-B,TYR & Castanospermine & Infection \\
\hline$\underline{I L 2 R A}$ & $\begin{array}{l}\text { basiliximab| daclizumab, } \\
\text { Zenapax|Denileukin diftitox|aldesleukin }\end{array}$ & $\begin{array}{l}\text { Immunosuppression| For prophylactic treatment of } \\
\text { kidney transplant rejection| treatment of cutaneous } \\
\text { nT-cell lymphoma| treatment of adults with metastatic } \\
\text { renal cell carcinoma. }\end{array}$ \\
\hline IFIHI & Hyaluronan & $\begin{array}{l}\text { osteoarthritis|plastic } \\
\text { surgery|ophthalmology|interstitial cystitis }\end{array}$ \\
\hline ADPRH, HLA-B, IL2RA, SLCI A2,TG, TYR & Adenylate & $\begin{array}{l}\text { nutritional supplementation| dietary shortage or } \\
\text { imbalance }\end{array}$ \\
\hline ADPRH, IL2RA & Hydroxychloroquine & $\begin{array}{l}\text { malaria|discoid and systemic lupus erythematosus, and } \\
\text { rheumatoid arthritis. }\end{array}$ \\
\hline
\end{tabular}


Table Continued.....

\begin{tabular}{lll}
\hline Vitiliogo associated genes (VAG) & Drugs (drug bank compounds) & Indications \\
\hline GZMB, HLA-B, IL2RA, TG,TYR & Paraffin & $\begin{array}{l}\text { emulsifying agent for cosmetic creams and lotions, } \\
\text { mineral oil and paraffin wax emulsions, as a biological } \\
\text { buffer, and used as an alkalizer. }\end{array}$ \\
CASP7, GZMB, HLA-B, IL2RA, TG & Cyclosporin A & $\begin{array}{l}\text { treatment of transplant (kidney, liver, and heart) } \\
\text { rejection, rheumatoid arthritis, severe psoriasis. } \\
\text { chronic myelogenous (myeloid, myelocytic, } \\
\text { granulocytic) leukemia|immunosuppressive effect on }\end{array}$ \\
ADPRH, HLA-B & Busulfan & bone marrow. \\
ADPRH, CASP7,TG & Adp ribose & \\
ADPRH,IYR & Chitosan & cosmetic \\
GZMB, HLA-B, IL2RA,TICAMI & Rantes & acute and chronic inflammation \\
ADPRH, ATXN2, RERE, SLCIA2,TG & Glutamine & Nutraceutical \\
GZMB, TG & Chromium & Nutraceutical \\
CASP7, FANCA, IL2RA, TG,TYR & Cisplatin|5FU|Methotrexate & Anticancer \\
SLC44A4 & Choline & Nutraceutical| CNS disorders \\
TYR & Azelaic & Acid|Monobenzone|Mimosine|NADH
\end{tabular}

The drug bank compounds encompassing FDA approved drugs and Nutraceuticals indications for the VAG are shown. Skin indications are bolded. FDA drug targets are underlined.

Abbreviations: FDA, federal drug administration; IVIG, intravenous immunoglobulin; RANTES, regulated on activation, normal T cell expressed and secretedCCL5

In addition, other approved drugs implicated with the Vitiligo associated genes included 1) anti-neoplastics (Hydrogen peroxide, Concanomycin A, Phycoerythrin, basiliximab, daclizumab, Zenapax, Denileukin diftitox, aldesleukin, Busulfan, Cisplatin|5FU|Methotrexate), 2) antiinfectives (Hydrogen peroxide, Concanamycin A, Castanospermine, Hydroxychloroquine), 3) antiinflammatory (Intravenous immunoglobulin, Rantes), rheumatoid arthritis (Hydroxychloroquine, Cyclosporin A) and 4) Nutraceticals and supplements (Aspartate, Arginine, Glutamate, Glucosamine, Adenylate, Chromium, Choline, Polysaccharide, chondroitin sulfate). Of particular interest was the discovery of Vitiligo genes associated with dermatological applications including (ADP-ribosylarginine hydrolase |ADPRH; caspase 7 |CASP7; granzyme B |GZMB; major histocompatibility complex, class I, B $\mid$ HLA-B; solute carrier family 1 (glial high affinity glutamate transporter), member 2 ISLC1A2; thyroglobulin |TG and tyrosinase TYR; interferon induced with helicase $\mathrm{C}$ domain 1 |IFIH1 and interleukin 2 receptor, alpha|IL2RA).

\section{Discussion}

Vitiligo, a complex acquired skin disorder, has been known for centuries. ${ }^{4}$ Various theories including autoimmune, inflammation, melanocytes, neuronal, stress and environmental factors have been put forward over the last decade..$^{3,6,7,9}$ The cause and etiology, however, remain unclear. It is likely that a combination of factors is responsible in susceptible individuals. The disease, while not life threatening, affects a large number of people around the world (1\%) across racial divide, gender and ages. ${ }^{1,17}$ The psychological trauma associated with Vitiligo exerts a considerable toll on the patients in view of visible skin abnormality particularly in exposed areas such as the face. ${ }^{16}$

Currently a cure remains elusive and treatment options are very limited and their effects often only temporary. In fact, the only FDA approved treatment is the use of UV, 308nm Excimer Laser. ${ }^{28}$ Other treatment options include use of topical calcineurin inhibitors, steroids and vitamins, phototherapy with UV-B, oral antioxidants, herbal supplements and nutraceuticals, depigmentation and camouflaging using cosmetics. ${ }^{3,5,17,35}$ These treatments have varying degrees of success and the disease remains unconquered. Encouragingly, a number of clinical trials are underway (Supplemental Table S1). Discovery of novel targets for druggableness and off label use of existing drugs is an attractive option to tackle this disease. Experience is emerging for repurposing existing, FDA approved drugs for other diseases. ${ }^{36,62,67}$ Since these drugs are already in the clinic and their level of toxicity is known, it is relatively easy to develop off the label use for at least some of the drugs, if a molecular link is demonstrable.

In recent years, a huge amount of genetic information has been generated using the GWAS approach. ${ }^{68-73}$ With the recent advances in human protein expression datasets ${ }^{47,49,57}$ protein $3 \mathrm{D}$ structure availability for the majority of human proteins, and with the use of powerful chemogenomics tools to predict druggableness, ${ }^{42,74-76}$ accelerated drug discovery starting from the genome is becoming a reality.

Reasoning that a working database of genes associated with Vitiligo can be an attractive starting point for novel diagnostics and therapeutics, the GWAS datasets were mined for VAG (Supplemental Table S1). Fifty-one genes showing polymorphic genetic association with Vitiligo encompassing protein coding and non-coding sequences were identified in this study. Encouraged by the preliminary results that the VAG included putative druggable targets including enzymes, transporters, transcription factors and receptors, ${ }^{58}$ a comprehensive analysis was undertaken.

Two of the 51 genes (Tyrosinase, TYR and Interleukin-2 receptor subunit alpha, IL2RA) are on the FDA approved target list. ${ }^{46,57}$ The IL2RA targeted drugs are immune modulators, which inhibit 
cytokines production and reduce pro inflammatory cytokines; this imples a strong possibility of therapeutic advantage for Vitiligo. The second FDA approved target, TYR targeted drugs, includes anti neoplastics, antibiotics, anti-inflammatory, antioxidants, neurotrophic, neutraceuticals and vitamins. The strong Vitiligo association seen with these two approved targets and the plethora of drugs and nutraceuticals available against these targets provide a strong rationale for testing for off label use.

The genes associated with Vitiligo were also found to be associated with various autoimmune diseases by the NCBI PheGenI tool (Systemic lupus, multiple scelerosis, diabetes Type I, hypothyroidism, rheumatoid arthritis) as well as with cancer, inflammation, infections and neurological disorders. These results are consistent with the prevailing theories that imply diverse etiology. ${ }^{1,3,6,7,17,55}$ In addition, pathway mapping from the Gene Analytics meta analysis tool also provided corroborating evidence for the involvement of immune, infection, endocrine, apoptosis, NF-kB transcription factor signaling, metabolic perturbation and neuronal pathways. Specific as well as common genes from the VAG are implicated with these pathways. Drugs implicated in these pathways may provide valuable additions to the therapy of Vitiligo. In particular, drugs targeting NF-KB transcription factor, diverse apoptotic caspase-related cascade inhibitors, cytokine signaling inhibitors and inhibitors of neuronal pathways including Dopamine, Parkinson's and neurotransmitter uptake inhibitors, may be of therapeutic benefit to the Vitiligo patients.

While diagnosis of Vitiligo is relatively easy due to the visible skin abnormalities (white patches), prediction of risk of development of the disease, in particular at a early age as well as monitoring a response to therapy urgently need genetic markers that can be easily monitored. To this end, secreted proteins in body fluids (the secretome) offer a biomarker potential. Half of the VAG proteins are detected in the body fluids (25/51). Five of these secreted proteins were also detected in the skin tissue (ADP-ribosylarginine hydrolase |ADPRH, DEAD (Asp-Glu-Ala-Asp) box helicase 6| DDX6, LIM domain containing preferred translocation partner in lipoma| LPP, ribonuclease T2| RNASET2 and thyroglobulin |TG). In particular, the proteins detected in the body fluids such as ear wax, saliva, semen, milk and urine offer a means to develop noninvasive biomarkers; the proteins detected in the blood and serum can provide a less invasive detection capability. If a definitive link with Vitiligo is established for these biomarkers, they could provide a response to therapy potential for dermatologists to follow. Protein-based nanochips can be easily developed to simultaneously monitor these biomarkers. ${ }^{77}$

The current GWAS data allowed for segregation of VAG linked polymorphic variants across diverse human DNAs (Chinese/Korean/ Japanese; Asian Indians, Saudi; Hispanics; Africans and Caucasians and Europeans). Unique as well as common VAG markers were identified in the study. The unique markers included protein coding sequences as well as pseudogenes and ncRNAs. These markers deserve additional studies and currently ongoing diverse clinical trials could benefit by understanding the expression profile of these genes. Numerous drug-like compounds were identified in the study using chemogenomics approaches. These compounds were predicted to be nontoxic (lack of toxicophore), orally bioavailable (RO5 value zero) and bioactive at $\mathrm{nM}$ concentrations. Five lead targets and bioactive compounds were identified in the study (caspase 7, apoptosis-related cysteine peptidase $\mid$ CASP7, fibroblast activation protein, alpha| FAP, granzyme B (granzyme 2, cytotoxic T-lymphocyte-associated serine esterase 1) $\mid$ GZMB, solute carrier family 1 (glial high affinity glutamate transporter), member 2 |SLC1A2 and tyrosinase (TYR) with a high degree of selectivity for the compounds; two of the inhibitors were highly selective to the target protein (GZMB and TYR). These leads can be rapidly taken to animal models ${ }^{78,79}$ for efficacy and toxicity testing and may provide a rationale for pharmaceutical drug development. As a backup, 363 bioactive compounds were discovered in the study. These compounds expand the drug discovery pipeline for the treatment of Vitiligo. The results provide further support for the concept of accelerated drug discovery approaches starting from the GWAS data by using chemoinformatics approaches. While a cure for Vitiligo may be far away, it is possible to develop a rationale for repurposing drugs currently approved for other indications for the treatment of Vitiligo. While previously the pharmaceutical industry was reluctant to take this approach, it is becoming increasingly attractive. Such repurposing is applicable not only for already approved drugs, but also for failed drugs. ${ }^{67}$ The great advantage of such an approach is that the toxicity, side effects and bioavailability information is already available from the FDA database. The genes associated with Vitiligo were found to be associated with numerous approved drugs and nutraceuticals. These drugs are linked to treatment of cancer, inflammation, autoimmune diseases, immunosuppression, infections, arthritis, transplant rejection and skin disorders including psoriasis. Thus, these drugs can readily be tested in mouse models and in the clinic either as a single agent or in combination with other treatments such as phototherapy or Excimer laser. At the very least, these drugs could help fill the void that currently exists for the treatment of Vitiligo.

\section{Summary}

The Genome Wide Association datasets provided an attractive starting point for gene target discovery for the skin disease Vitiligo. Use of chemoinformatics and chemogenomics approaches led to the identification of druggable targets, bioactive compounds, FDA approved drugs and nutraceuticals associated with the Vitiligo genome. In view of the serious shortage of drugs for Vitiligo, which affects over $1 \%$ of the global population, the drugs identified in this study can be easily repurposed for the treatment of Vitiligo.

\section{Acknowledgements}

This work was supported in part by the Genomics of Cancer Fund, Florida Atlantic University Foundation. I thank the CanSar gene annotation tool for valuable datasets. I also thank Jeanine Narayanan for editorial assistance.

\section{Conflict of interest}

The author declares no conflict of interest.

\section{References}

1. Allam M, Riad H. Concise review of recent studies in Vitiligo. Qatar Med J. 2013;(2):1-19.

2. Taïeb A, Picardo M. Clinical practice: Vitiligo. $N$ Engln $\mathrm{J}$ Med. 2009;360(2):160-169.

3. Ghafourian A, Ghafourian S, Sadeghifard N, et al. Vitiligo: symptoms, pathogenesis and treatment. Int $J$ Immunopathol Pharmacol. 2014;27(4):485-489.

4. Millington GW, Levell NJ. Vitiligo: the historical curse of depigmentation. Int J Dermatol. 2007;46(9):990-995.

5. Bacigalupi RM, Postolova A, Davis RS. Evidence-based, non-surgical treatments for vitiligo: a review. Am J Clin Dermatol. 2012;13(4):217237. 
6. Boissy RE, Spritz RA. Frontiers and controversies in the pathobiology of vitiligo: separating the wheat from the chaff. Exp Dermatol. 2009;18(7):583-585.

7. Czajkowski R, Mecinska-Jundzill K. Current aspects of vitiligo genetics. Postepy Dermatol Alergol. 2014;31(4):247-255.

8. Glassman SJ. Vitiligo, reactive oxygen species and T-cells. Clin Sci (Lond). 2011;120(3):99-120.

9. Guerra L, Dellambra E, Brescia S, et al. Vitiligo: pathogenetic hypotheses and targets for current therapies. Curr Drug Metab. 2010;11(5):451-467.

10. Laddha NC, Dwivedi M, Mansuri MS, et al. Vitiligo: interplay between oxidative stress and immune system. Exp Dermatol. 2013;22(4):245250.

11. Miniati A, Weng Z, Zhang B, et al. Neuro-immuno-endocrine processes in vitiligo pathogenesis. Int J Immunopathol Pharmacol. 2012;25(1):1-7.

12. Mosenson JA, Eby JM, Hernandez C, et al. A central role for inducible heat-shock protein 70 in autoimmune vitiligo. Exp Dermatol. 2013;22(9):566-559.

13. Tarle RG, Nascimento LM, Mira MT, et al. Vitiligo - part 1. An Bras Dermatol. 2014;89(3):461-470.

14. Spritz RA. The genetics of generalized vitiligo: autoimmune pathways and an inverse relationship with malignant melanoma. Genome medicine. 2010;2(10):78.

15. Parsad D, Dogra S, Kanwar AJ. Quality of life in patients with vitiligo. Health Qual Life Outcomes. 2003;1:58.

16. Teovska Mitrevska N, Eleftheriadou V, Guarneri F. Quality of life in vitiligo patients. Dermatol Ther. 2012;25(Suppl 1):S28-S31.

17. Abu Tahir M, Pramod K, Ansari SH, et al. Current remedies for vitiligo. Autoimmun Rev. 2010;9(7):516-520.

18. Anbar TS, Hegazy RA, Picardo M, et al. Beyond vitiligo guidelines: combined stratified/personalized approaches for the vitiligo patient. Exp Dermatol. 2014;23(4):219-223.

19. Cavalie M, Ezzedine K, Fontas E, et al. Maintenance therapy of adult vitiligo with $0.1 \%$ tacrolimus ointment: a randomized, double blind, placebo-controlled study. J invest dermatol. 2015;135(4):970-994.

20. Szczurko O, Shear N, Taddio A, et al. Ginkgo biloba for the treatment of vitilgo vulgaris: an open label pilot clinical trial. BMC Complement Altern Med. 2011;11:21.

21. Parsad D, Pandhi R, Juneja A. Effectiveness of oral Ginkgo biloba in treating limited, slowly spreading vitiligo. Clin Exp Dermatol. $2003 ; 28(3): 285-287$.

22. Garg T, Chander R, Jain A. Combination of microdermabrasion and 5fluorouracil to induce repigmentation in vitiligo: an observational study. Dermatol Surg. 2011;37(12):1763-1766.

23. Srinivas CR, Shenoi SD, Balachandran C. Acceleration of repigmentation in vitiligo by topical minoxidil in patients on photochemotherapy. Int $J$ Dermatol. 1990;29(2):154-155.

24. Camacho F, Mazuecos J. Oral and topical L-phenylalanine, clobetasol propionate, and UVA/sunlight — a new study for the treatment of vitiligo. J Drugs Dermato. 2002;1(2):127-131.

25. Narahari SR, Ryan TJ, Bose KS, et al. Integrating modern dermatology and Ayurveda in the treatment of vitiligo and lymphedema in India. Int $J$ Dermatol. 2011;50(3):310-334.

26. Hariharan V, Klarquist J, Reust MJ, et al. Monobenzyl ether of hydroquinone and 4-tertiary butyl phenol activate markedly different physiological responses in melanocytes: relevance to skin depigmentation. $J$ invest dermatol. 2010;30(1):211-220.
27. Mouzakis JA, Liu S, Cohen G. Rapid response of facial vitiligo to $308 \mathrm{~nm}$ excimer laser and topical calcipotriene. J Clin Aesthet Dermatol. 2011;4(6):41-44.

28. Spencer JM, Nossa R, Ajmeri J. Treatment of vitiligo with the 308-nm excimer laser: a pilot study. J Am Acad Dermatol. 2002;46(5):727-731.

29. Birlea SA, Gowan K, Fain PR, et al. Genome-wide association study of generalized vitiligo in an isolated European founder population identifies SMOC2, in close proximity to IDDM8. $J$ invest dermatol. 2010;130(3):798-803.

30. Birlea SA, Jin Y, Bennett DC, et al. Comprehensive association analysis of candidate genes for generalized vitiligo supports XBP1, FOXP3, and TSLP. $J$ invest dermatol. 2011;131(2):371-381.

31. Jin Y, Birlea SA, Fain PR, et al. Genome-wide association analyses identify 13 new susceptibility loci for generalized vitiligo. Nature genetics. 2012;44(6):676-680.

32. Jin Y, Birlea SA, Fain PR, et al. Variant of TYR and autoimmunity susceptibility loci in generalized vitiligo. $N$ Engl $J$ med. 2010;362(18):1686-1697.

33. Spritz RA. Six decades of vitiligo genetics: genome-wide studies provide insights into autoimmune pathogenesis. $J$ invest dermatol. 2012;32(2):268-273.

34. Cheong KA, Kim NH, Noh M, et al. Three new single nucleotide polymorphisms identified by a genome-wide association study in Korean patients with vitiligo. J Korean Med Sci. 2013;28(5):775-779.

35. Narayanan R. Ebola-associated genes in the human genome: Implications for novel targets. MOJ Proteomics Bioinform. 2014;1(5):00032.

36. Narayanan R. Druggableness of the Ebola Associated Genes in the Human Genome: Chemoinformatics Approach. MOJ Proteomics Bioinform. 2015;2(2):00038.

37. Narayanan R. Druggable Cancer Secretome: Neoplasm-associated Traits. Cancer genomics \& proteomics. 2015;12(3):119-131.

38. Welter D, MacArthur J, Morales J, et al. The NHGRI GWAS Catalog, a curated resource of SNP-trait associations. Nucleic acids research. 2014;42(Database issue):D1001-D1006.

39. Ramos EM, Hoffman D, Junkins HA, et al. Phenotype-Genotype Integrator (PheGenI): synthesizing genome-wide association study (GWAS) data with existing genomic resources. Eur J Hum Genet. 2014;22(1):144-147.

40. Safran M, Dalah I, Alexander J, et al. GeneCards Version 3:the human gene integrator. Database (Oxford). 2010;2010:baq020.

41. Halling-Brown MD, Bulusu KC, Patel M, et al. canSAR: an integrated cancer public translational research and drug discovery resource. Nucleic acids research. 2012;40(Database issue):D947-D956.

42. Bulusu KC, Tym JE, Coker EA, et al. canSAR:updated cancer research and drug discovery knowledgebase. Nucleic acids research. 2014;42(Database issue):D1040-D1047.

43. Bento AP, Gaulton A, Hersey A, et al. The ChEMBL bioactivity database:an update. Nucleic acids research. 2014;42(Database issue):D1083-D1090.

44. Huang DW, Sherman BT, Lempicki RA. Systematic and integrative analysis of large gene lists using DAVID bioinformatics resources. Nat Protocols. 2008;4(1):44-57.

45. Magrane M, Consortium U. UniProt Knowledgebase: a hub of integrated protein data. Database (Oxford). 2011;2011:bar009.

46. Law V, Knox C, Djoumbou Y, et al. DrugBank 4.0: shedding new light on drug metabolism. Nucl acids res. 2014;42(Database issue):D1091D1097. 
47. Kim MS, Pinto SM, Getnet D, et al. A draft map of the human proteome Nature. 2014;509(7502):575-581.

48. Wilhelm M, Schlegl J, Hahne H, et al. Mass-spectrometry-based draft of the human proteome. Nature. 2014;509(7502):582-587.

49. Kolker E, Higdon R, Haynes W, et al. MOPED: Model Organism Protein Expression Database. Nucleic acids research. 2012;40(Database issue):D1093-D1099.

50. Montague E, Stanberry L, Higdon R, et al. MOPED 2.5-an integrated multi-omics resource: multi-omics profiling expression database now includes transcriptomics data. Omics. 2014;18(6):335-343.

51. Lipinski CA, Lombardo F, Dominy BW, et al. Experimental and computational approaches to estimate solubility and permeability in drug discovery and development settings. Adv drug deliv rev. 2001;46(13):3-26.

52. Lipinski CA. Lead- and drug-like compounds: the rule-of-five revolution. Drug Discov Today Technol. 2004;1(4):337-341.

53. Oprea TI, Davis AM, Teague SJ, et al. Is there a difference between leads and drugs? A historical perspective. J chem inf comput sci. 2001;41(5):1308-1315.

54. Williams DP, Naisbitt DJ. Toxicophores: groups and metabolic routes associated with increased safety risk. Curr opin drug discov \& devel. 2002;5(1):104-115.

55. Colucci R, Lotti T, Moretti S. Vitiligo: an update on current pharmacotherapy and future directions. Expert Opin Pharmacother. 2012;13(13):1885-1899.

56. Consortium GP. A map of human genome variation from populationscale sequencing. Nature. 2010;467(7319):1061-1073.

57. Uhlen M, Fagerberg L, Hallstrom BM, et al. Proteomics. Tissue-based map of the human proteome. Science. 2015;347(6220):1260419.

58. Hopkins AL, Groom CR. The druggable genome. Nat rev Drug discov. 2002;1(9):727-730.

59. Stastna M, Van Eyk JE. Investigating the Secretome: Lessons About the Cells That Comprise the Heart. Cir Cardiovasc Genet. 2012;(1):o8-o18.

60. Sioud M, Leirdal M. Druggable signaling proteins. Methods mol biol. 2007;361:1-24.

61. Dixon SJ, Stockwell BR. Identifying druggable disease-modifying gene products. Curr opin chem biol. 2009;3(5-6):549-555.

62. Mullard A. Drug repurposing programmes get lift off. Nat rev Drug discov. 2012;11(7):505-506.

63. Shim JS, Liu JO. Recent advances in drug repositioning for the discovery of new anticancer drugs. Int J Biol Sci. 2014;10(7):654-663.

64. Lencz T, Malhotra AK. Targeting the schizophrenia genome: a fast track strategy from GWAS to clinic. Mol psychiatry. 2015;20(7):820-826.
65. Kouznetsova J, Sun W, Martinez-Romero C, et al. Identification of 53 compounds that block Ebola virus-like particle entry via a repurposing screen of approved drugs. Emerg Microbes Infect. 2014;3(12):e84.

66. Rask-Andersen M, Masuram S, Schioth HB. The druggable genome: Evaluation of drug targets in clinical trials suggests major shifts in molecular class and indication. Annu Rev Pharmacol Toxicol. 2014;54:9 26.

67. Jin G, Wong ST. Toward better drug repositioning: prioritizing and integrating existing methods into efficient pipelines. Drug discovery today. 2014;19(5):637-644.

68. Denny JC, Bastarache L, Ritchie MD, et al. Systematic comparison of phenome-wide association study of electronic medical record data and genome-wide association study data. Nat Biotechnol. 2013;31(12):11021111.

69. Gottesman O, Kuivaniemi H, Tromp G, et al. The Electronic Medical Records and Genomics (eMERGE) Network: past, present, and future. Genet med. 2013;15(10):761-771.

70. Postmus I, Trompet S, Deshmukh HA, et al. Pharmacogenetic metaanalysis of genome-wide association studies of LDL cholesterol response to statins. Nat commun. 2014;5:5068.

71. Shungin D, Winkler TW, Croteau-Chonka DC, et al. New genetic loci link adipose and insulin biology to body fat distribution. Nature. 2015;518(7538):187-196.

72. Simon-Sanchez J, Singleton A. Genome-wide association studies in neurological disorders. Lancet Neurol. 2008;7(11):1067-1072.

73. Wolpin BM, Rizzato C, Kraft P, et al. Genome-wide association study identifies multiple susceptibility loci for pancreatic cancer. Nature genetics. 2014;46(9):994-1000.

74. Flight MH. Chemogenomics: A change of tactic. Nature reviews Drug discovery. 2008;7(2):122.

75. Gaulton A, Bellis LJ, Bento AP, et al. ChEMBL: a large-scale bioactivity database for drug discovery. Nucleic acids research. 2012;40(Database issue):D1100-D1107.

76. Jacoby E. Computational chemogenomics. Wiley Interdisciplinary Reviews: Computational Molecular Science. 2011;1(1):57-67.

77. Christodoulides N, Pierre FN, Sanchez X, et al. Programmable bionanochip technology for the diagnosis of cardiovascular disease at the point-of-care. Methodist Debakey Cardiovasc J. 2012;8(1):6-12.

78. Harris JE, Harris TH, Weninger W, et al. A mouse model of vitiligo with focused epidermal depigmentation requires IFN-gamma for autoreactive CD8(+) T-cell accumulation in the skin. J invest dermatol. 2012;132(7):1869-1876.

79. Smyth JR Jr, McNeil M. Alopecia areata and universalis in the Smyth chicken model for spontaneous autoimmune vitiligo. J Investig Dermatol Symp Proc. 1999;4(3):211-215. 